\title{
Article \\ The UV-B-Induced Transcription Factor HY5 Regulated Anthocyanin Biosynthesis in Zanthoxylum bungeanum
}

\author{
Jing Zhou ${ }^{1, \dagger}$, Jiaxin Meng ${ }^{1,+}$, Shuangyu Zhang ${ }^{1}$, Rufei Chi ${ }^{1}$, Cheng Wang ${ }^{2}$, Dongmei Wang ${ }^{2, *}$ and Houhua Li $^{1, *}$ \\ 1 College of Landscape Architecture Sand Art, Northwest A\&F University, Xianyang 712100, China; \\ zjmmxx@nwafu.edu.cn (J.Z.); mengjiaxin26@nwafu.edu.cn (J.M.); zhangshungyu@nwafu.edu.cn (S.Z.); \\ chirufei@nwafu.edu.cn (R.C.) \\ 2 College of Forestry, Northwest A\&F University, Xianyang 712100, China; jmcookie@nwsuaf.edu.cn \\ * Correspondence: dmwang@nwafu.edu.cn (D.W.); lihouhua@nwafu.edu.cn (H.L.) \\ $\dagger$ These authors contributed equally to this work.
}

Citation: Zhou, J.; Meng, J.; Zhang, S.; Chi, R.; Wang, C.; Wang, D.; Li, H The UV-B-Induced Transcription Factor HY5 Regulated Anthocyanin Biosynthesis in Zanthoxylum bungeanum. Int. J. Mol. Sci. 2022, 23 2651. https://doi.org/10.3390/ ijms23052651

Academic Editor: Karen Skriver

Received: 31 December 2021

Accepted: 23 February 2022

Published: 28 February 2022

Publisher's Note: MDPI stays neutral with regard to jurisdictional claims in published maps and institutional affiliations.

Copyright: (C) 2022 by the authors. Licensee MDPI, Basel, Switzerland. This article is an open access article distributed under the terms and conditions of the Creative Commons Attribution (CC BY) license (https:// creativecommons.org/licenses/by/ $4.0 /)$

\begin{abstract}
Pericarp color is an important economic characteristic of Zanthoxylum bungeanum. Anthocyanins are the main reason for the pericarp's red appearance in Z. bungeanum. In this study, through the combined analysis of the metabolome and transcriptome, HY5, whose expression is highly correlated to changes in the anthocyanin content, was screened and identified. Under natural ripening conditions, the Z bungeanum fruit gradually changed in color from green to red, while bagging resulted in the fruit maintaining its green color. After unbagging, the fruit gradually turned red, and the ZbHY5 expression and anthocyanin content increased. In addition, the leaves changed from green to red after exposure to UV-B radiation, and the ZbHY5 expression and anthocyanin content increased. The transient overexpression of ZbHY5 deepened the redness of the Z. bungeanum leaves and promoted the expression of $Z b H Y 5$ and $Z b M Y B 113$ as well as anthocyanin accumulation. Bimolecular fluorescence complementation (BIFC) showed that there was an interaction between $\mathrm{ZbHY} 5$ and ZbMYB113. These results revealed that under UV-B irradiation, ZbHY5 might regulate the expression levels of the structural genes related to anthocyanin biosynthesis through combination with ZbMYB113, thereby affecting anthocyanin accumulation. This finding provides useful insights for further studies focusing on UV-B-induced anthocyanin accumulation in Z. bungeanum.
\end{abstract}

Keywords: Zanthoxylum bungeanum; anthocyanins; UV-B; ZbHY5; ZbMYB113

\section{Introduction}

Zanthoxylum bungeanum (Rutaceae family) has a long cultivation and application history of nearly three thousand years in China. The brilliant red pericarp color of $Z$. bungeanum, which is greatly appreciated as a traditional condiment by consumers, is due to anthocyanins, water-soluble flavonoid pigments. Anthocyanins are widely found in the flowers, stems, leaves, fruits, and other tissues of plants and are one of the main causes of plant color presentation. Additionally, anthocyanins are potentially beneficial to human health and have demonstrated anti-cancer, anti-inflammatory, anti-aging, and anti-obesity effects; improvements in brain function; and eyesight and cardiovascular protection, etc. They have also been recognized as important antioxidants [1].

Phenylalanine is the direct precursor of anthocyanins and other flavonoids. It is converted to $\rho$-coumaroyl CoA via the action of the phenylalanine lyase (PAL), cinnamate 4-hydroxylase $(\mathrm{C} 4 \mathrm{H})$ and 4-coumarate: $\mathrm{CoA}$ ligase (4CL). In general, anthocyanins are catalyzed from $\rho$-coumaroyl $\mathrm{CoA}$ and malonyl-CoA by a series of enzymes that are encoded by structural genes, which mainly include the early biosynthesis genes (EBGs) CHS (Chalcone synthase gene), $\mathrm{CHI}$ (Chalcone isomerase gene), and $\mathrm{F3H}$ (flavanone-3-hydroxylase gene), and the late biosynthesis genes (LBGs) $F 3^{\prime} H$ (flavonoid $3^{\prime}$-hydroxylase gene), $F 3^{\prime} 5^{\prime} H$ (flavonoid 3',5'-hydroxylase gene), DFR (dihydroflavonol-4-reductase gene), ANS (anthocyanin synthase gene), and UFGT (flavonoid 3-O-glucosyltransferase gene) [2]. In addition, 
the transcription of these structural genes is controlled by regulatory factors, including by the members of protein families that contain R2R3-MYB domains, bHLH (basic helix-loophelix) domains, and conserved WD40 repeat transcription factors (TFs) [3]. For example, MYB113, an R2R3-MYB gene, has been proven to be involved in anthocyanin synthesis in plants such as A. thaliana and Pistacia chinensis [4,5]. Additionally, bHLH35 was shown to regulate anthocyanin synthesis at high temperatures in Solanum melongena [6]. In recent years, other transcription factors have also been found to be involved in the anthocyanin synthesis, such as bZIP and WRKY. A recent study found that WRKY co-regulates the anthocyanin pathway synthesis with the MBW complex (the transcriptional complex of the MYB, bHLH, and WD40 proteins) [7].

Light is an important environmental factor that can regulate various plant growth and development processes, such as seed germination, morphogenesis, shade response, flowering, and senescence. Light is not only an energy source for photosynthesis, but it is also a biological stimulus that is present throughout the life cycle of plants, triggering cellular responses through specific sensory photoreceptors [8]. The current research has shown that, in many plants, the light signal transduction pathways are able to regulate anthocyanin accumulation to a certain extent $[9,10]$. In Arabidopsis thaliana, some lightinduced transcription factor genes can promote anthocyanin biosynthesis, such as $M Y B$ (AtMYB75 and AtMYB90) and bHLH (AtTT8, AtGL3 and AtEGL3) [11]. In strawberry (Fragaria $\times$ ananassa) fruit, the transient overexpression of the light-induced FvbHLH9 can promote the accumulation of anthocyanins, while the interference of FvbHLH9 can reduce anthocyanin accumulation [12]. In recent years, in addition to MYB, bHLH, and WD40, some other transcription factors were also found to be involved in anthocyanin synthesis. Among them, ELONGATED HYPOCOTYL 5 (HY5) is the most critical and representative transcription factor in plant photomorphogenesis and plays a significant role in the regulation of anthocyanin biosynthesis [13]. It should be noted that the production of other flavonoids was also affected by light. HY5 promoted flavonol accumulation through the activation of MYB12 expression in A. thaliana [14].

HY5 is a basic leucine zipper (bZIP) transcription factor. The HY5 protein was shown to experience degradation in dark environments; providing UV-B prevented this degradation, HY5 gene expression was reactivated, and HY5 protein was accumulated once again [15]. In Arabidopsis thaliana, it was found that HY5 can target a large quantity of light-responsive genes in vivo. This is mainly due to the typical bZIP domain of HY5, which can directly bind to the G-box (CACGTG) element or ACGT-containing elements (ACEs) of their promoters [16]. In addition, in vitro and in vivo analyses have shown that HY5 acts as a positive regulator in the anthocyanin accumulation process by directly binding to the promoters of structural genes during anthocyanin biosynthesis [17]. In addition, MYB113 was found to interact with HY5 to positively regulate anthocyanin biosynthesis in $A$. thaliana and Malus domestica [18].

In this study, a bZIP transcription factor ZbHY5 was isolated from Z. bungeanum. Bioinformatics analysis and transient gene overexpression confirmed that $Z b H Y 5$ played a positive regulatory role in the anthocyanin biosynthesis of Z. bungeanum. Additionally, through BIFC, we found that there was an interaction between ZbHY5 and ZbMYB113. This study verified that $H Y 5$ was involved in the regulation of $Z$. bungeanum anthocyanin biosynthesis, providing a theoretical reference for the light-induced anthocyanin accumulation of Z. bungeanum.

\section{Results}

\subsection{Analyses of Anthocyanin Composition and Content during the Ripening of Z. bungeanum Fruit}

The metabolome data showed that eight anthocyanins were detected during the four Z. bungeanum fruit-ripening stages: Cyanidin-3-O-galactoside, Cyanidin-3-O-glucoside (Kuromanin), Cyanidin-3-O-rutinoside (Keracyanin), Peonidin-3-O-glucoside, Delphinidin3-O-(6"-O-acetyl) glucoside, Pelargonidin-3-O-rutinoside, Pelargonidin-3,5-O-diglucoside, and Cyanidin-3-O-galactoside. Among them, the Cyanidin-3-O-galactoside content was 
the highest, and the Cyanidin-3,5-O-diglucoside (Cyanin) content was the second highest. Specifically, the total anthocyanin content increased throughout the four stages (Table 1).

Table 1. Anthocyanin composition and content during the ripening of Z. bungeanum fruit.

\begin{tabular}{|c|c|c|c|c|}
\hline Anthocyanins & S1 & S2 & S3 & S4 \\
\hline Cyanidin-3-O-galactoside & $(1.57 \pm 0.02) \times 10^{7} \mathrm{~d}$ & $(1.06 \pm 0.02) \times 10^{8} \mathrm{c}$ & $(9.07 \pm 0.29) \times 10^{7} \mathrm{~b}$ & $(1.25 \pm 0.02) \times 10^{8} \mathrm{a}$ \\
\hline $\begin{array}{l}\text { Cyanidin-3-O-glucoside } \\
\text { (Kuromanin) }\end{array}$ & $(1.71 \pm 0.04) \times 10^{6} \mathrm{~d}$ & $(9.87 \pm 0.4) \times 10^{6} \mathrm{c}$ & $(2.85 \pm 0.01) \times 10^{7} \mathrm{~b}$ & $(6.18 \pm 0.22) \times 10^{7} \mathrm{a}$ \\
\hline $\begin{array}{l}\text { Cyanidin-3-O-rutinoside } \\
\text { (Keracyanin) }\end{array}$ & $(6.27 \pm 0.13) \times 10^{5} \mathrm{a}$ & $(3.96 \pm 0.04) \times 10^{5} \mathrm{~b}$ & $(3.12 \pm 0.02) \times 10^{5} \mathrm{c}$ & $(3.33 \pm 0.06) \times 10^{5} \mathrm{c}$ \\
\hline Peonidin-3-O-glucoside & $(1.92 \pm 0.19) \times 10^{3} \mathrm{a}$ & $(3.42 \pm 0.54) \times 10^{3} \mathrm{a}$ & $(3.24 \pm 1.70) \times 10^{3} \mathrm{a}$ & $(1.81 \pm 0.34) \times 10^{3} \mathrm{a}$ \\
\hline $\begin{array}{l}\text { Delphinidin-3-O-(6"-O-acetyl) } \\
\text { glucoside }\end{array}$ & $(1.12 \pm 0.03) \times 10^{6} \mathrm{a}$ & $(7.90 \pm 0.20) \times 10^{5} \mathrm{~b}$ & $(4.58 \pm 0.19) \times 10^{5} \mathrm{c}$ & $(3.50 \pm 0.65) \times 10^{5} \mathrm{c}$ \\
\hline Pelargonidin-3-O-rutinoside & $(2.19 \pm 0.04) \times 10^{5} \mathrm{a}$ & $(1.41 \pm 0.02) \times 10^{5} \mathrm{~b}$ & $(1.00 \pm 0.03) \times 10^{5} \mathrm{c}$ & $(8.11 \pm 0.38) \times 10^{4} \mathrm{~d}$ \\
\hline Pelargonidin-3,5-O-diglucoside & $(8.01 \pm 0.45) \times 10^{4} \mathrm{a}$ & $(3.33 \pm 0.02) \times 10^{4} \mathrm{~b}$ & $(2.37 \pm 0.51) \times 10^{4} \mathrm{c}$ & $(3.76 \pm 0.43) \times 10^{4} \mathrm{~b}$ \\
\hline $\begin{array}{c}\text { Cyanidin-3,5-O-diglucoside } \\
\text { (Cyanin) }\end{array}$ & $(1.71 \pm 0.02) \times 10^{7} \mathrm{~d}$ & $(1.15 \pm 0.01) \times 10^{8} \mathrm{~b}$ & $(9.46 \pm 0.17) \times 10^{7} \mathrm{c}$ & $(1.36 \pm 0.03) \times 10^{8} \mathrm{a}$ \\
\hline Total anthocyanins & $(3.65 \pm 0.03) \times 10^{7} \mathrm{~d}$ & $(2.32 \pm 0.03) \times 10^{8} \mathrm{c}$ & $(2.15 \pm 0.05) \times 10^{8} \mathrm{~b}$ & $(3.23 \pm 0.03) \times 10^{8} \mathrm{a}$ \\
\hline
\end{tabular}

S1-S4 represent the four gradual ripening stages of Z. bungeanum fruit: S1 (green stage), S2 (light red stage), S3 (red stage), and S4 (deep red stage). Different lowercase letters indicate significant differences among treatments according to one-way ANOVA test $(p<0.05)$.

2.2. Analysis of Expression Levels of Anthocyanin Biosynthesis-Related Genes during the Ripening of Z. bungeanum Fruit

The transcriptome data showed that during the Z. bungeanum fruit-ripening process, the $Z b H Y 5$ and $Z b M Y B 113$ expression levels gradually increased. The structural genes related to anthocyanin biosynthesis ( $\mathrm{ZbCHS}, \mathrm{ZbCHI}, \mathrm{ZbDFR}, \mathrm{ZbANS}$ ) followed the expression pattern of these transcription factors with a short delay. The ZbDFR expression level at $S 4$ was only slightly above the level at S1, while $\mathrm{ZbCHS}, \mathrm{ZbCHI}$ and $\mathrm{ZbANS}$ increased 4.6-, 1.6-, and 2.9-fold, respectively. This suggested that different target genes were activated with different strengths. HY5 is a bZIP transcription factor that plays a key role in anthocyanin biosynthesis. ZbMYB113, whose homologous gene AtMYB113 was also reported to be important in anthocyanin biosynthesis, presented the same change trend with ZbHY5 (Table 2).

Table 2. Expression levels of anthocyanin biosynthesis-related genes during the ripening of $Z$. bungeanum fruit.

\begin{tabular}{ccccc}
\hline Gene Name & S1 & S2 & S3 & S4 \\
\hline ZbHY5 & $66.33 \pm 1.23 \mathrm{~b}$ & $153.74 \pm 6.13 \mathrm{a}$ & $172.40 \pm 14.95 \mathrm{a}$ & $189.11 \pm 2.17 \mathrm{a}$ \\
ZbMYB113 & $1.83 \pm 0.65 \mathrm{c}$ & $4.45 \pm 1.21 \mathrm{c}$ & $19.71 \pm 0.85 \mathrm{~b}$ & $57.59 \pm 2.73 \mathrm{a}$ \\
ZbbHLH35 & $20.65 \pm 1.02 \mathrm{~b}$ & $41.08 \pm 3.93 \mathrm{a}$ & $25.51 \pm 1.24 \mathrm{~b}$ & $29.41 \pm 2.65 \mathrm{~b}$ \\
ZbCHS & $160.66 \pm 2.56 \mathrm{~b}$ & $147.64 \pm 3.25 \mathrm{~b}$ & $435.95 \pm 2.47 \mathrm{~b}$ & $746.46 \pm 34.78 \mathrm{a}$ \\
ZbCHI & $69.74 \pm 1.84 \mathrm{c}$ & $45.62 \pm 2.92 \mathrm{~d}$ & $104.60 \pm 3.88 \mathrm{~b}$ & $115.01 \pm 2.12 \mathrm{a}$ \\
ZbF3H & $465.99 \pm 6.53 \mathrm{bc}$ & $439.16 \pm 5.81 \mathrm{c}$ & $483.21 \pm 12.77 \mathrm{~b}$ & $314.87 \pm 13.38 \mathrm{a}$ \\
ZbDFR & $46.28 \pm 0.50 \mathrm{~b}$ & $10.30 \pm 0.66 \mathrm{~d}$ & $20.39 \pm 0.16 \mathrm{c}$ & $52.18 \pm 1.77 \mathrm{a}$ \\
ZbANS & $52.82 \pm 4.49 \mathrm{c}$ & $41.63 \pm 1.43 \mathrm{c}$ & $75.15 \pm 3.46 \mathrm{~b}$ & $151.92 \pm 9.12 \mathrm{a}$ \\
ZbUFGT & $36.74 \pm 0.59 \mathrm{~d}$ & $84.75 \pm 3.92 \mathrm{~b}$ & $103.24 \pm 4.06 \mathrm{a}$ & $84.03 \pm 1.41 \mathrm{c}$ \\
\hline
\end{tabular}

S1-S4 represent the four gradual ripening stages of Z. bungeanum fruit: S1 (green stage), S2 (light red stage), S3 (red stage), and S4 (deep red stage). Different lowercase letters indicate significant differences among treatments according to one-way ANOVA test $(p<0.05)$.

\subsection{Phylogenetic Tree Analysis of HY5 and Other bZIP Family Transcription Factors in Z. bungeanum}

Through a keyword search, local blastp alignment, a local hmmer search, and the deletion of sequences whose domains were incomplete, 47 target sequences were finally obtained as bZIP family protein sequences of Z. bungeanum, which were named ZbbZIP01—47 
according to their ID numbers in the transcriptome. Wolfgang et al. (2018) updated the classification method of the bZIP transcription factor family in the model plant Arabidopsis thaliana in 2018 [19]. They divided the bZIP transcription factor of Arabidopsis thaliana into 12 subfamilies, which were labeled from A to K, and S. Zhang et al. (2011) studied Arabidopsis thaliana and found that HY5 and HYH in the H subfamily were involved in anthocyanin synthesis [20]. Through the analysis of the phylogenetic relationship with Arabidopsis thaliana, it was found that there was only one $\mathrm{H}$ subfamily member in Z. bungeanumZbHY5 (ZbbZIP43) (Figure 1a). The analysis of the differential expression patterns showed that the expression level of $Z b H Y 5$ was relatively high throughout all stages and showed a gradual upward trend as the Z. bungeanum fruit grew and developed (Figure $1 b$ ). The correlation analysis heat map showed that the expression level of ZbHY5 was positively correlated with the total anthocyanin content and that the correlation coefficient was as high as 0.93 (Figure 1c). These results proved that we successfully screened ZbHY5, the key transcription factor gene involved in anthocyanin synthesis, from the bZIP family of Z. bungeanum.

\subsection{Clone and Identification of HY5 in Z. bungeanum}

In Arabidopsis thaliana and some other plants, HY5 plays an important role in anthocyanin biosynthesis [16,21]. To verify the function of HY5 in Z. bungeanum, the HY5 gene was cloned from $Z$. bungeanum leaves. The gene sequencing results showed that the HY5 had a 504-bp ORF, which encoded a protein containing 167 amino acids. Multiple sequence alignments showed that the C-terminus of HY5 contained a bZIP domain (amino acids 90-141) (Figure 2a). This was designated as ZbHY5.

The phylogenetic tree of HY5 in Z. bungeanum and the other 42 plants showed that ZbHY5 had the highest homology with CcHY5 (Citrus clementina) (Figure 2b). Loyola et al. proved that HY5 plays an important role in the transmission of light signals and the regulation of anthocyanin accumulation in grapes [22]. Huang et al. found that HY5 is involved in anthocyanin synthesis in some citrus plants, such as in blood orange and purple pummelo. These results revealed that ZbHY5 may be related to anthocyanin regulation in Z. bungeanum and provided a basis for further research [23].

\subsection{Bagging Inhibited Anthocyanin Synthesis in Z. bungeanum Fruit}

In the fruit that had been taken out of the bags at 0 days $(0 \mathrm{~d}), 10$ days $(10 \mathrm{~d})$, and 20 days ( $20 \mathrm{~d}$ ), the color of the pericarp gradually changed from green to red. Bagging, on the one hand, delayed the pericarp coloring process and resulted in the color of the bagged fruit being significantly lighter than that of the fruit exposed to light (Figure 3a).

The results of the total anthocyanin content determination were consistent with the phenotypic changes that we observed. The total anthocyanin content in the pericarp showed a gradual upward trend from $0 \mathrm{~d}$ to $10 \mathrm{~d}$, and it then increased rapidly in the $20 \mathrm{~d}$ fruit (Figure $3 b$ ). This suggests that anthocyanin accumulation is delayed to a certain extent after bagging and that the anthocyanin content would eventually be reduced.

Compared to $0 \mathrm{~d}$, the expression levels of the transcription factor genes (ZbHY5, ZbMYB113, ZbbHLH35) and the structural genes (ZbCHS, ZbCHI, ZbF3H, ZbDFR, ZbANS, ZbUFGT) related to anthocyanin synthesis in the $10 \mathrm{~d}$ and $20 \mathrm{~d}$ fruits increased significantly. However, compared to $10 \mathrm{~d}$, only the expression levels of $\mathrm{ZbCHS}, \mathrm{ZbF} 3 \mathrm{H}, \mathrm{ZbDFR}, \mathrm{ZbANS}$, and ZbUFGT increased in the $20 \mathrm{~d}$ fruit, while the expression levels of the other genes (ZbHY5, ZbMYB113, ZbbHLH35, and ZbCHI) decreased slightly (Figure 3c). This might be due to the sudden exposure to light after long-term shading increasing the expression levels of genes in response to sharp increases in the amount of light and allowing the genes to achieve their maximum expression in a short time, exceeding the expression levels of these genes in the $20 \mathrm{~d}$ samples. These results revealed that light could significantly promote anthocyanin accumulation in Z. bungeanum by regulating the expression of related genes. 


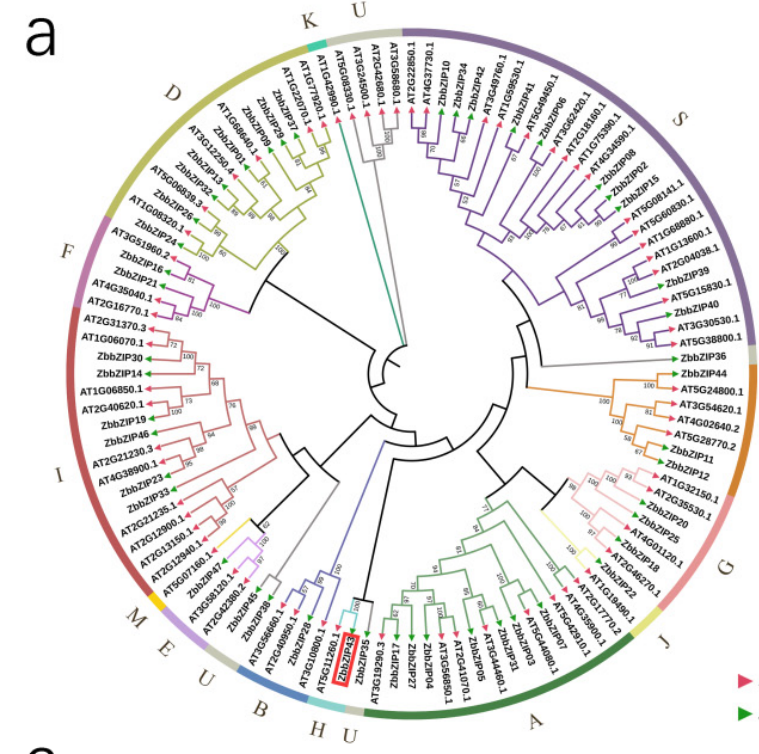

C

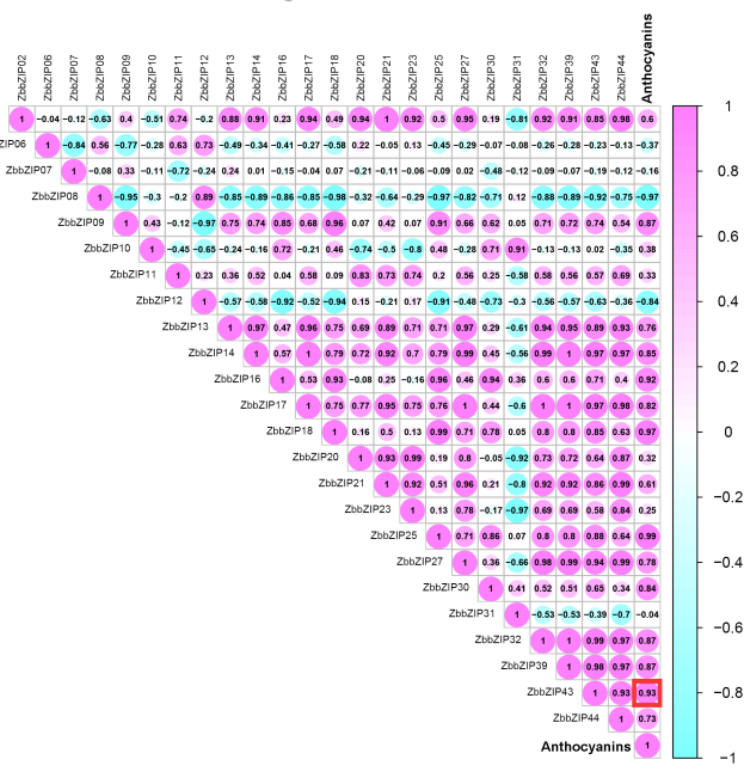

b
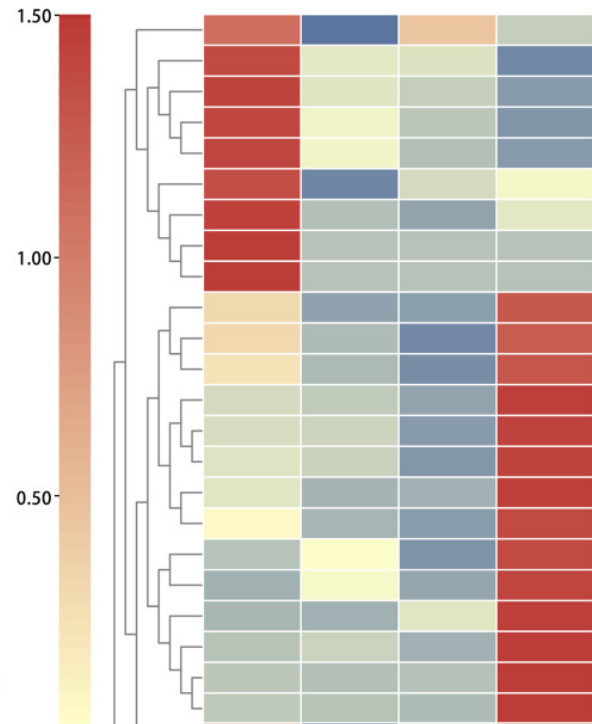

bZIP06 S

ZbbZIP12 C

ZbbZIP47 E ZbbZIP19 (I)

ZbbZIP46 (1) ZbbZIP40 S ZbbZIP45 U ZbbZIP34 S ZbbZIP38 U ZbbZIP37 D ZbbZIP02 S ZbbZIP21 F ZbbZIP17 A ZbbZIP27 A ZbbZIP28 B ZbbZIP42 S ZbbZIP44 C ZbbZIP13 D ZbbZIP29 D ZbbZIP43 H ZbbZIP14 (I ZbbZIP32 D ZbbZIP39 $\mathrm{S}$ ZbbZIP11 C ZbbZIP20 G ZbbZIP04 A ZbbZIP23 I ZbbZIP30 (I) ZbbZIP09 D ZbbZIP16 F ZbbZIP18 G ZbbZIP25 G ZbbZIP33 1 ZbbZIP22 J ZbbZIP10 S ZbbZIP41 S ZbbZIP07 A ZbbZIP35 U ZbbZIP03 A ZbbZIP26 D ZbbZIP05 A ZbbZIP31 A ZbbZIP01 D ZbbZIP08 S ZbbZIP24 D ZbbZIP15 S ZbbZIP36 U

$-1.50$

Figure 1. Phylogenetic tree and correlation analysis of HY5 and other bZIP family transcription factors in Z. bungeanum. (a) Phylogenetic tree of ZbbZIP transcription factor family. The phylogenetic tree was constructed using the neighbor joining (NJ) method. The ZbHY5 candidate, ZbbZIP43, is marked by the red box. (b) Heat map of bZIP transcription factor gene expression in Z. bungeanum fruits at different growth and development stages. ZbbZIP43 is marked by the red box. S1-S4 represent the four gradual ripening stages of Z. bungeanum fruit: S1 (green stage), S2 (light red stage), S3 (red stage), and S4 (deep red stage). (c) Heat map representing the correlation between bZIP transcription factor gene expression and the anthocyanin content in the different growth and development stages of Z. bungeanum fruit. The number in the box represents the correlation between the abscissa and the ordinate. The positive value represents a positive correlation, the negative value represents a negative correlation, and the larger the absolute value of the number is, the stronger the correlation is. The 0.93 marked by the red box shows that there was a positive correlation between the anthocyanin content and ZbbZIP43 expression, and the correlation coefficient was 0.93 . 
a

ZbHY5

CCHY5

ATHY5

VvHY5

VrHY5

CsHY5

CqHY5-like

ZjHY5-like

CmHY5-like

RCHY 5

QSHY5

QSHY5

Consensus

ZbHY 5

CCHY 5

ATHY 5

VvHY 5

VrHY5

CSHY 5

CqHY5-like

SOHY 5

ZjHY5-like

CmHY5-like

RcHY5

QSHY5

Q.SHY5

Consensus

ZbHY 5

CCHY5

ATHY5

VvHY5

VrHY 5

CsHY 5

CqHY5-like

SOHY 5

zjHY5-like

CmHY5-like

RcHY 5

QSHY 5

QsHY 5

Consensus

b
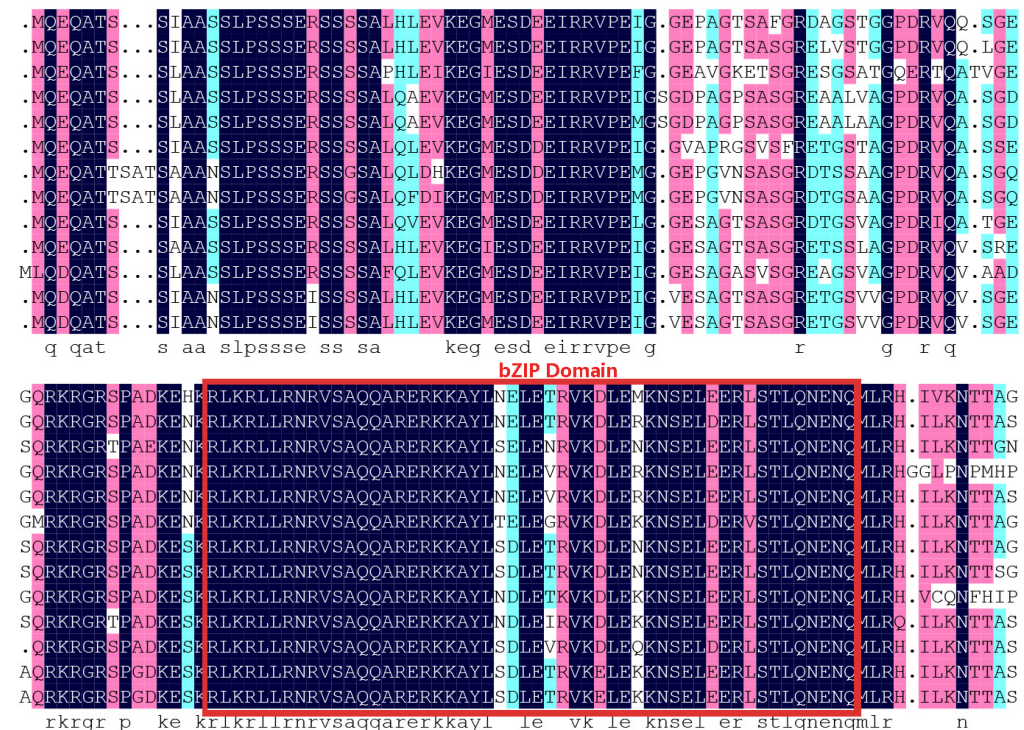

53

154

155

154

156

156

153

153
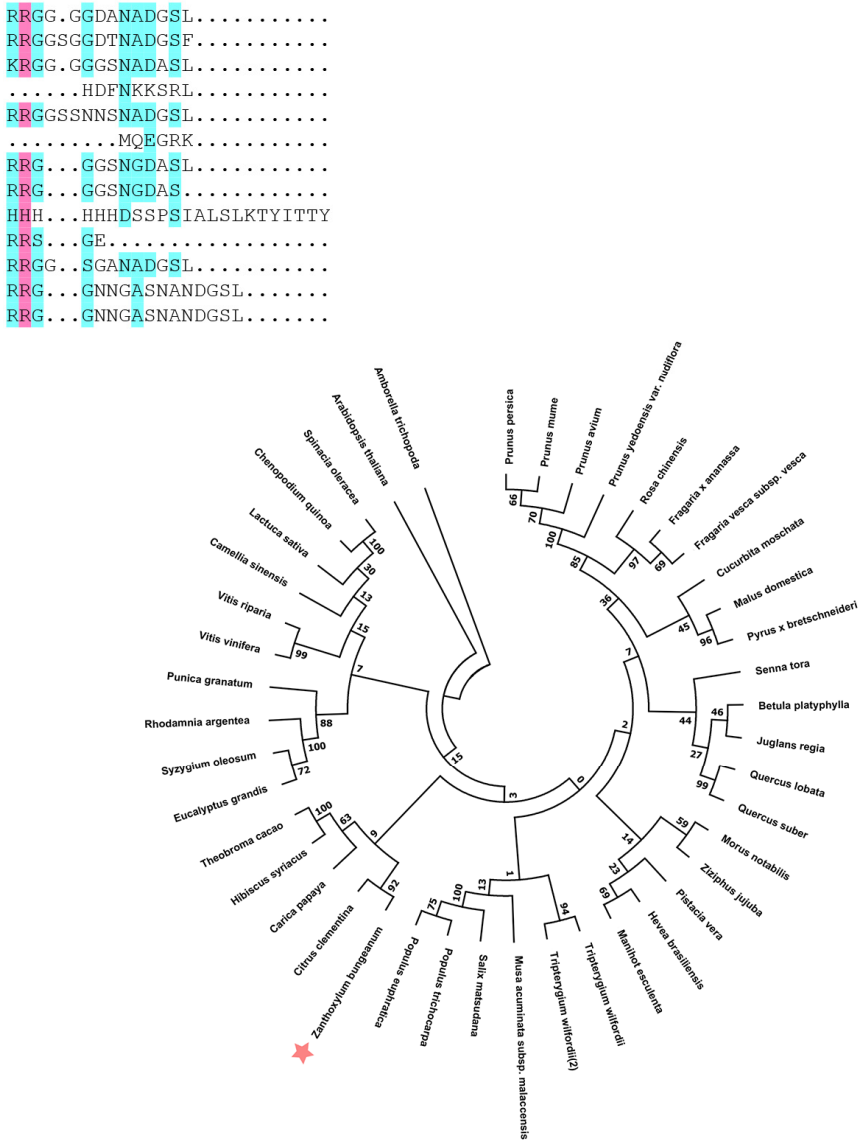

Figure 2. Sequence alignment and phylogenetic analysis of ZbHY5. (a) Protein alignment of ZbHY5 and its homologs, where the bZIP domain is marked with a red box. The phylogenetic tree was constructed using the neighbor joining (NJ) method. (b) Phylogenetic analysis of ZbHY5 and 42 other plants. Zanthoxylum bungeanum (ZbHY5) is denoted by a red asterisk. Arabidopsis thaliana, BDQV01000026.1; Betula platyphylla, AHY20043.1; Citrus clementina, XP_006450470.1; Cucurbita moschata, XP_022962152.1; Carica papaya, XP_021891410.1; Chenopodium quinoa, XP_021758181.1; Camellia sinensis, XP_028120847.1; Eucalyptus grandis, XP_010048982.1; Fragaria $x$ ananassa, AKG58815.1; Fragaria vesca subsp. Vesca, XP_004291469.1; Hevea brasiliensis, XP_021670969.1; Hibiscus syriacus, XP_039039624.1; Juglans regia, XP_035548793.1; Lactuca sativa, XP_023759627.1; Musa acuminata 
subsp. Malaccensis, XP_009381123.1; Malus domestica, NP_001280752.1; Manihot esculenta, XP_021597568.1; Morus notabilis, XP_010110356.2; Prunus avium, XP_021827650.1; Pyrus $x$ bretschneideri, XP_009348603.1; Populus euphratica, XP_011039711.1; Punica granatum, XP_031407456.1; Prunus mume, XP_008219477.1; Prunus persica, XP_020411091.1; Populus trichocarpa, XP_002308656.1; Pistacia vera, XP_031286333.1; Prunus yedoensis var. Nudiflora, PQM36064.1; Quercus lobata, XP_030951444.1; Quercus suber, XP_023893213.1; Rhodamnia argentea, XP_030548349.1; Rosa chinensis, XP_024165510.1; Salix matsudana, QEH62726.1; Spinacia oleracea, XP_021837612.1; Senna tora, KAF7817238.1; Syzygium oleosum, XP_030440254.1; Theobroma cacao, XP_007013841.2; Tripterygium wilfordii, KAF5743305.1; Tripterygium wilfordii, XP_038698890.1; Vitis riparia, XP_034682512.1; Vitis vinifera, XP_010648648.1; Ziziphus jujuba, XP_024925020.1.

\subsection{UV-B Irradiation Promoted Anthocyanin Synthesis in Z. bungeanum Leaves}

After UV-B irradiation, the leaves of Z. bungeanum changed from green to red. No obvious changes were observed from 0 to $6 \mathrm{~h}$, but the leaves gradually turned red after $12 \mathrm{~h}$ (Figure 4a). Consistent with the phenotypic changes, total anthocyanin content in the leaves gradually increased from 0 to $24 \mathrm{~h}$ (Figure $4 \mathrm{~b}$ ). Interestingly, the qRT-PCR results showed that the expression levels of ZbHY5, ZbMYB113, ZbbHLH35, ZbCHS, ZbCHI, ZbF3H, ZbDFR, $Z b A N S$, and $Z b U F G T$ presented increasing and then decreasing trends. Among these genes, the expression levels of ZbHY5, ZbMYB113, ZbCHS, ZbCHI, ZbDFR, and ZbANS reached their maximum levels at $6 \mathrm{~h}$, while the expression levels of $Z b F 3 H$ and $Z b U F G T$ reached their maximum levels at $12 \mathrm{~h}$ (Figure 4c). These results indicated that UV-B promotes anthocyanin accumulation in the leaves of Z. bungeanum by controlling the expression of related genes.

\subsection{Transient Overexpression of ZbHY5 Promoted Anthocyanin Synthesis of Z. bungeanum Leaves}

Transient ZbHY5 overexpression was performed through infecting Z. bungeanum leaves with Agrobacterium tumefaciens containing empty pC2300 as the control and with Agrobacterium tumefaciens containing ZbHY5-pC2300, which served as the experimental group. After infection, the leaves were cultured in the dark for $24 \mathrm{~h}$ and then under UV-B irradiation for $18 \mathrm{~h}$. The leaves overexpressing ZbHY5 turned dark red, while the control leaves only showed a slight red coloration (Figure 5a). The total anthocyanin content in the leaves overexpressing ZbHY5 was significantly higher than that in the control leaves, with a 3.59-fold difference (Figure 5b). Additionally, further quantitative analysis found that the expression levels of ZbMYB113, ZbbHLH35, ZbCHS, ZbCHI, ZbF3H, ZbDFR, ZbANS, and $Z b U F G T$, which were related to anthocyanin biosynthesis in the leaves overexpressing ZbHY5, were also increased compared to the control 21.19-, 1.26-, 2.44-, 3.15-, 1.51-, 6.43-, 18.16-, and 2.06-fold, respectively (Figure 5c).

These results suggested that ZbHY5 could promote anthocyanin synthesis by inducing the expression of the key genes related to anthocyanin biosynthesis, leading to the accumulation of anthocyanin in the leaves of Z. bungeanum.

\subsection{ZbHY5 Can Interact with ZbMYB113 In Vivo}

In order to further study the anthocyanin synthesis regulation mechanism, the BiFC assay was performed to verify whether ZbHY5 could bind to ZbMYB113 to form a protein complex in vivo. Observation using a confocal laser scanning microscope revealed that only yellow fluorescence is observed in the nucleus of tobacco leaf cells when ZbHY5-YFPC and $\mathrm{ZbMYB113-YFP}$ are co-expressed. In contrast, there was no signal in the combinations of ZbMYB113-YFP $+Y P^{\mathrm{C}}$ or $\mathrm{YFP}^{\mathrm{N}}+\mathrm{ZbHY5-YFPC}$ (Figure 6). Therefore, the BiFC assay confirmed that ZbHY5 can interact with ZbMYB113. 
a

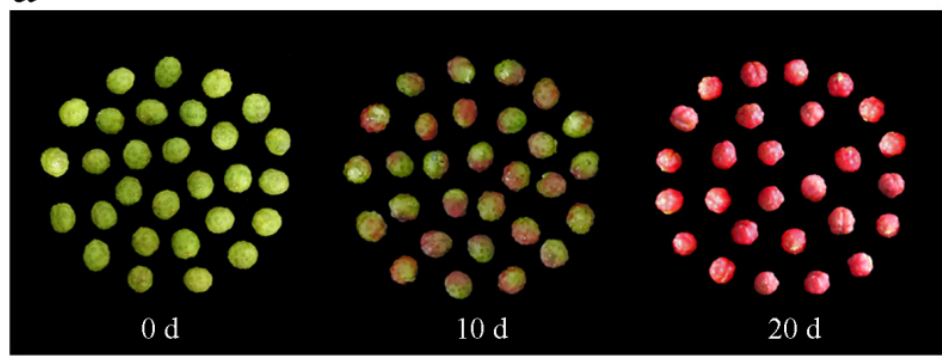

C

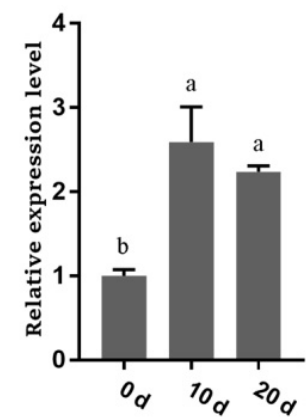

$\mathrm{ZbCHS}$

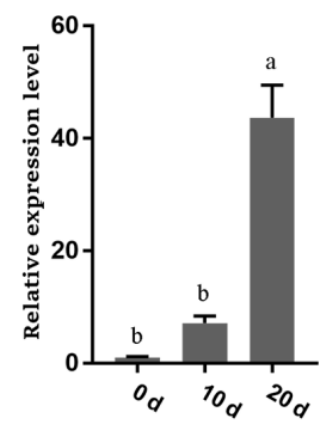

ZbDFR

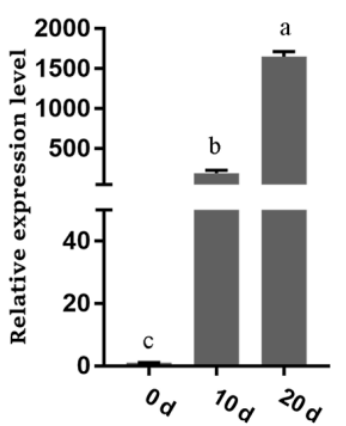

b

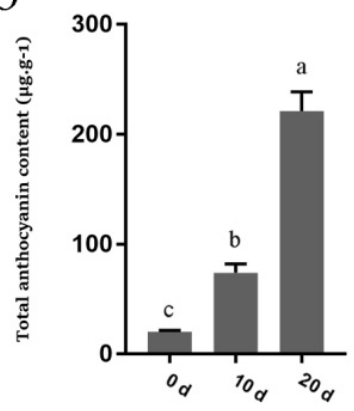

ZbMYB113

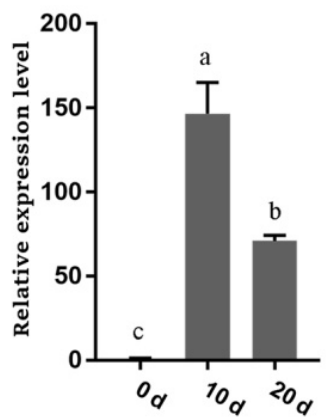

$\mathrm{ZbCHI}$

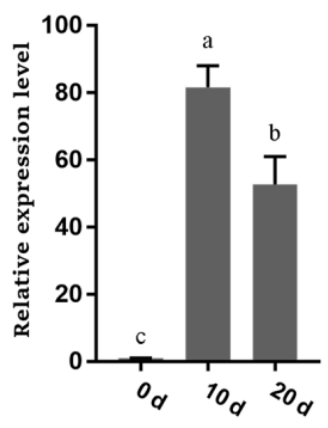

ZbANS

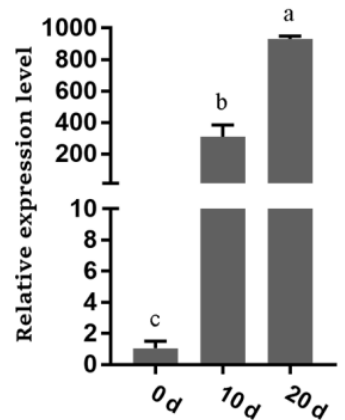

ZbbHLH35

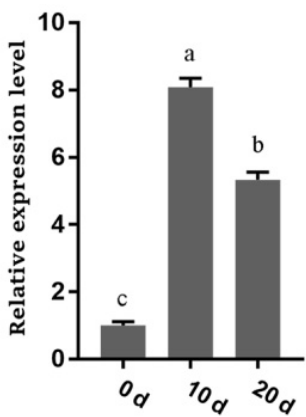

$\mathrm{ZbF} 3 \mathrm{H}$

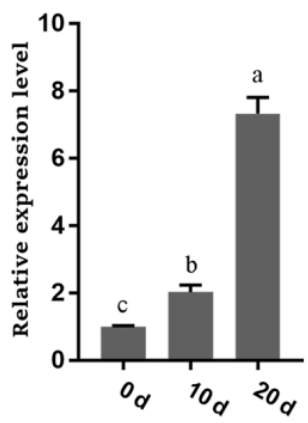

ZbUFGT

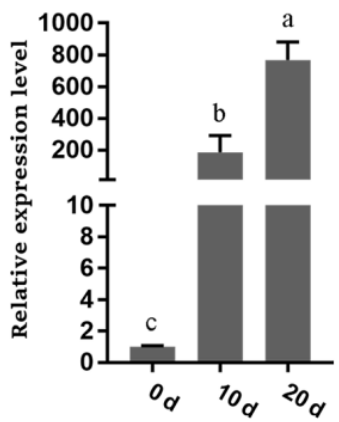

Figure 3. Total anthocyanin content and gene expression changes after the unbagging of Z. bungeanum fruit. (a) Pictures of Z. bungeanum fruit at 0 days $(0 \mathrm{~d}), 10$ days $(10 \mathrm{~d})$, and 20 days $(20 \mathrm{~d})$ after unbagging. (b) Total anthocyanin content in $0 \mathrm{~d}, 10 \mathrm{~d}$, and $20 \mathrm{~d}$ fruit. (c) The expression of the transcription factor genes (ZbHY5, ZbMYB113, ZbbHLH35) and structural genes (ZbCHS, ZbCHI, $\mathrm{ZbF3H}, \mathrm{ZbDFR}, \mathrm{ZbANS}, \mathrm{ZbUFGT}$ ) related to anthocyanin synthesis in $0 \mathrm{~d}, 10 \mathrm{~d}$, and $20 \mathrm{~d}$ fruit. Different lowercase letters indicate significant differences among treatments according to one-way ANOVA test $(p<0.05)$. 
a

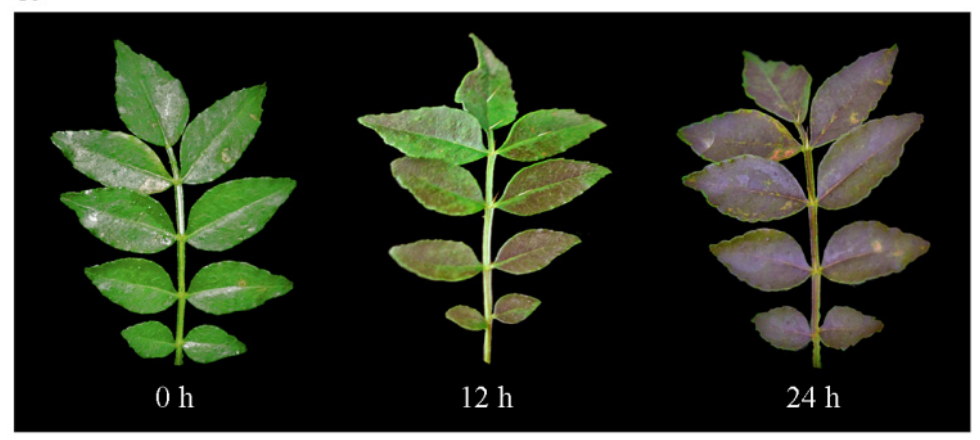

C

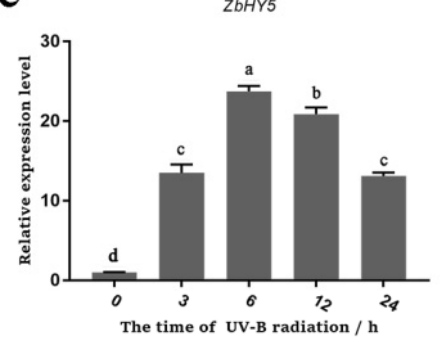

ZbCHS

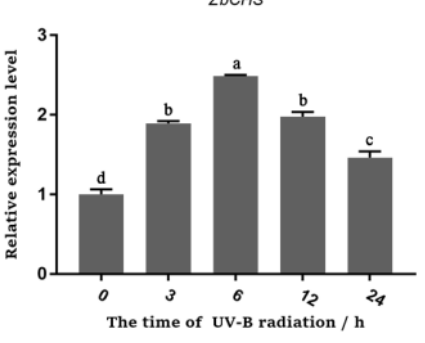

ZbDFR

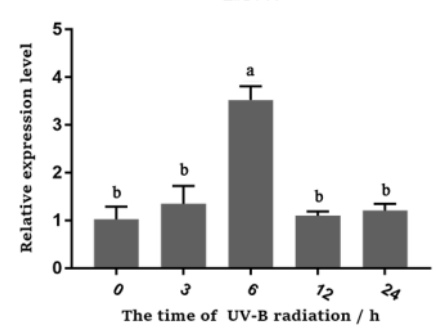

b

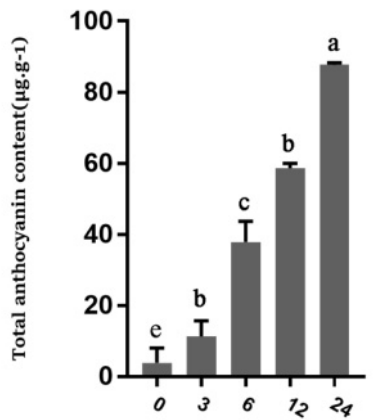

The time of UV-B radiation / $\mathrm{h}$

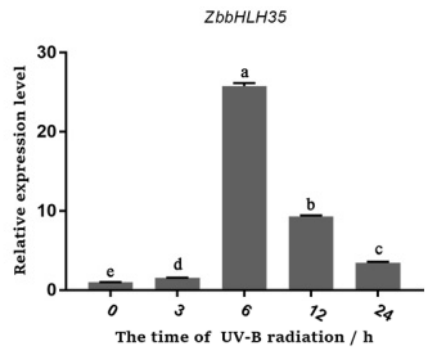

ZbF3H

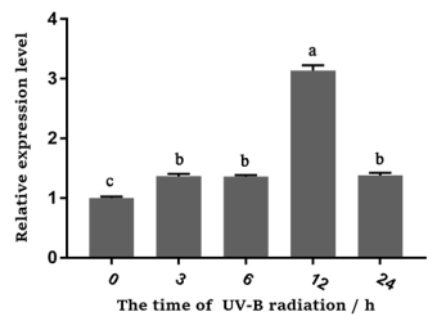

ZbUFGT

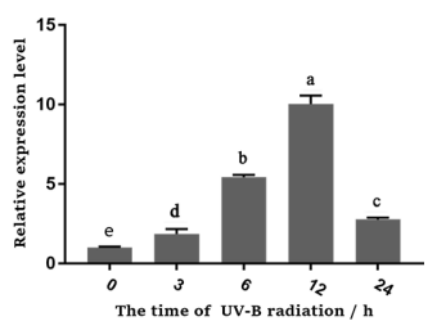

Figure 4. Changes in the total anthocyanin content and gene expression under UV-B irradiation in Z. bungeanum leaves. (a) Pictures of the Z. bungeanum leaves under UV-B irradiation after $0 \mathrm{~h}, 12 \mathrm{~h}$, and $24 \mathrm{~h}$. (b) The total anthocyanin content in the leaves after UV-B irradiation for $0 \mathrm{~h}, 3 \mathrm{~h}, 6 \mathrm{~h}, 12 \mathrm{~h}$, and $24 \mathrm{~h}$. (c) The expression of the transcription factor genes (ZbHY5, ZbMYB113, ZbbHLH35) and structural genes ( $\mathrm{ZbCHS}, \mathrm{ZbCHI}, \mathrm{ZbF3H}, \mathrm{ZbDFR}, \mathrm{ZbANS}, \mathrm{ZbUFGT}$ ) related to anthocyanin synthesis in the leaves after UV-B irradiation for $0 \mathrm{~h}, 3 \mathrm{~h}, 6 \mathrm{~h}, 12 \mathrm{~h}$, and $24 \mathrm{~h}$. Different lowercase letters indicate significant differences among treatments according to one-way ANOVA test $(p<0.05)$. 
a

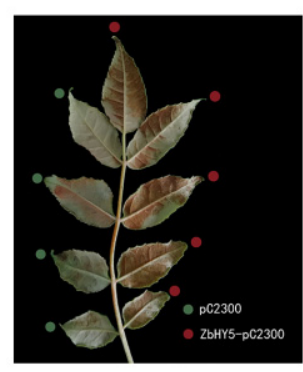

C
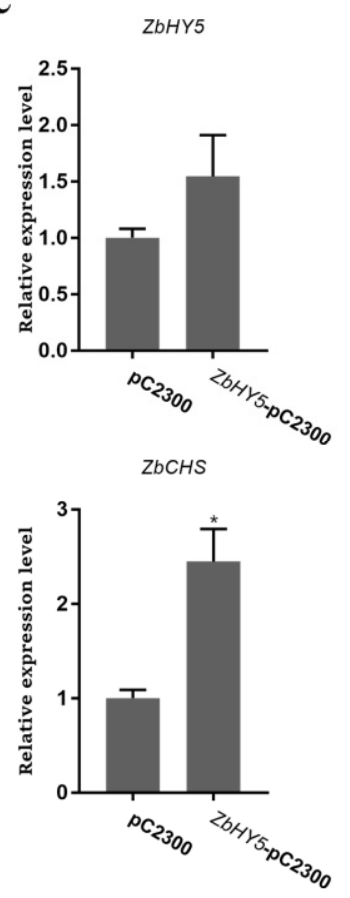

ZbDFR

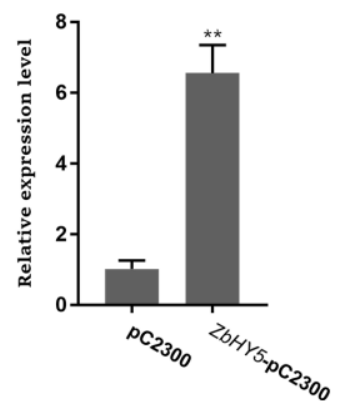

b

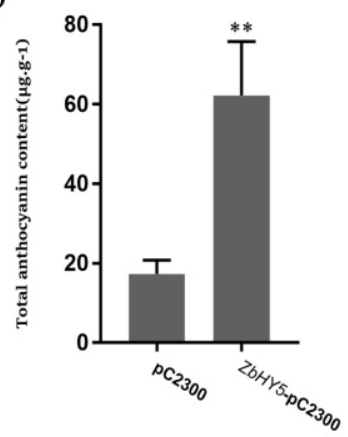

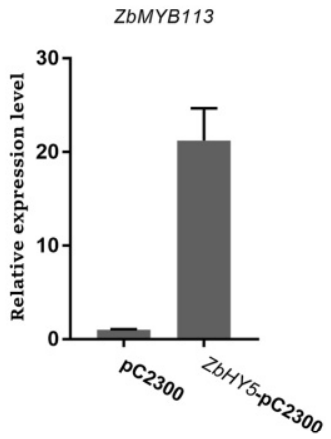

$\mathrm{ZbCHI}$

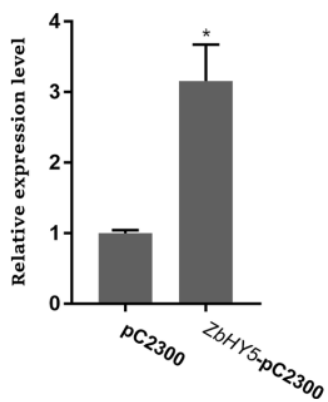

ZbANS

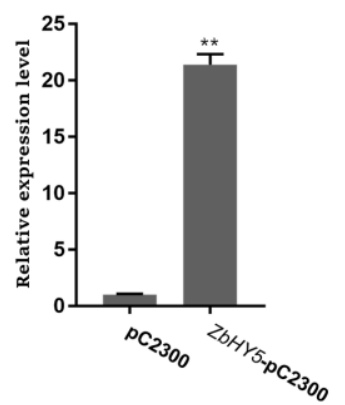

ZbbHLH35

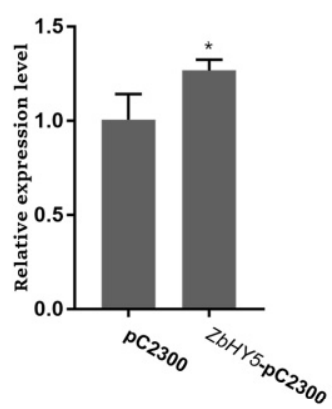

$\mathrm{ZbF} 3 \mathrm{H}$

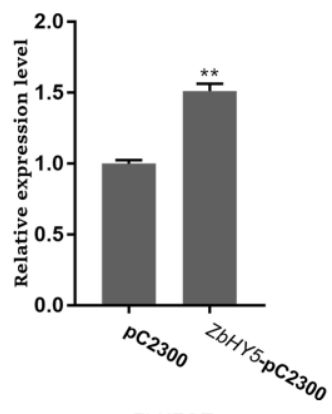

ZbUFGT

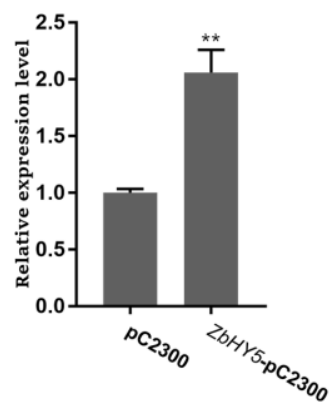

Figure 5. (a) Pictures of ZbHY5 gene overexpression phenotype changes in Z. bungeanum leaves. The green marks represent the control ( $\mathrm{pC} 2300)$ leaves, and the red marks represent the experimental group (ZbHY5-pC2300) leaves. (b) Determination of total anthocyanin content in ZbHY5 gene overexpression in Z. bungeanum leaves. (c) Quantitative analysis of genes related to ZbHY5 gene overexpression in the leaves. ${ }^{*} p<0.05,{ }^{* *} p<0.01$ using the $t$-test. 

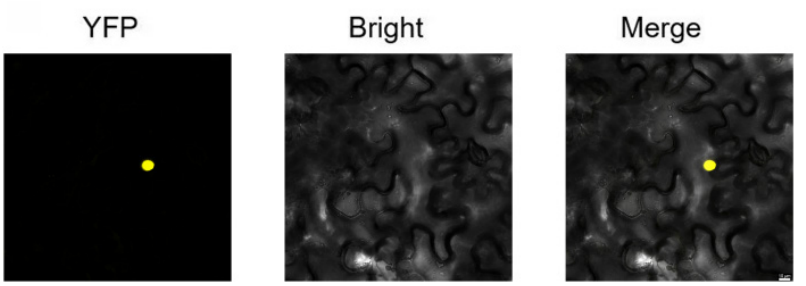

\author{
ZbMYB113-YFPN \\ $+$ \\ ZbHY5-YFPc
}
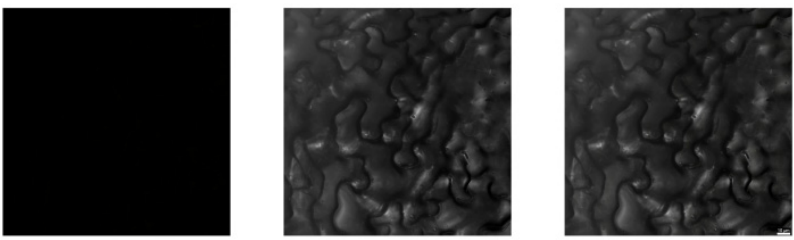
ZbMYB113-YFPN
$+$
YFPC
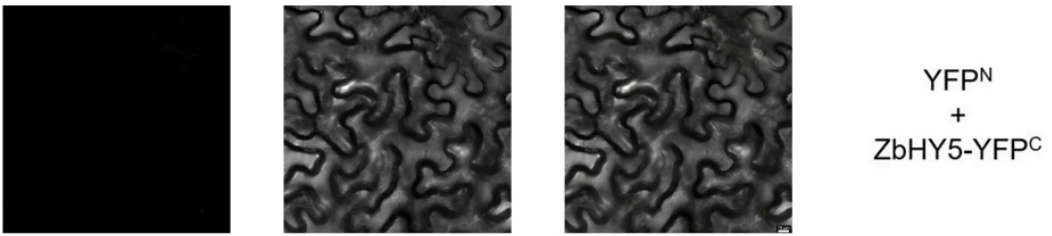

Figure 6. The BiFC assay was carried out to verify the interaction between ZbHY5 and ZbMYB113. Bars, $10 \mu \mathrm{m}$.

\title{
3. Discussion
}

For most plants, UV-B, the middle ultraviolet light $(\lambda=280-315 \mathrm{~nm})$, is an important factor for anthocyanin accumulation [24]. UV-B can promote anthocyanin accumulation because it promotes the expression of structural genes and regulatory genes in the anthocyanin biosynthesis pathway [11]. HY5, a bZIP transcription factor that participates in anthocyanin biosynthesis, is a downstream component of the light signaling pathway and is responsive to UV-B [25-27]. In Arabidopsis thaliana [14], Solanum lycopersicum [28], and blood orange (Citrus) [23], the HY5 protein was confirmed to participate in the anthocyanin synthesis process. In this experiment, the Z bungeanum fruit gradually changed from green to red under natural growth conditions because of anthocyanin accumulation, and the transcriptome data showed that the ZbHY5 expression level gradually increased. On the contrary, bagging blocked the fruit's reddening process, inhibited the expression of $Z b H Y 5$, and lessened the anthocyanin accumulation in Z. bungeanum fruit. In addition, under UV-B radiation, the leaves turned from green to red, from the ZbHY5 expression, and the anthocyanin content increased in the $Z$. bungeanum leaves. The transient overexpression of $Z b H Y 5$ in the leaves of $Z$. bungeanum caused significantly red coloration after $18 \mathrm{~h}$ of UV-B irradiation. The total anthocyanin content and the ZbHY5 expression levels increased significantly in the leaves overexpressing ZbHY5. These results verified that under UV-B irradiation, ZbHY5 played a key role in anthocyanin accumulation in Z. bungeanum.

HY5 can not only directly regulate the expression of the early and late structural genes in the downstream anthocyanin synthesis pathway [29], but it can also interact with other transcription factor proteins to indirectly regulate the late structural genes [30,31]. In Arabidopsis thaliana, HY5 bound itself directly to the PAP1 in vivo to regulate anthocyanin biosynthesis [32]. In Pyrus pyrifolia, HY5 physically interacted with MYB10 to positively regulate anthocyanin biosynthesis $[18,33]$. In this study, the transcription factor ZbMYB113 was screened and confirmed to be one of the R2R3-MYB activators involved in anthocyanin biosynthesis from $Z$. bungeanum. The transcriptome data showed that during the reddening of the Z. bungeanum fruit, ZbHY5 and ZbMYB113 expression levels gradually increased, and there was a high correlation between their expression levels. Bagging restrained the red process and the ZbHY5 and ZbMYB113 expression levels in Z. bungeanum fruit. Moreover, UV-B radiation made the leaves turn from green to red and promoted $Z b H Y 5$ and ZbMYB113 expression in Z. bungeanum. Furthermore, the overexpression of ZbHY5 
deepened the redness of the leaves and enhanced $Z b M Y B 113$ expression. Through the BiFC assay, we found that ZbMYB113 can interact with ZbHY5 in vivo. These results suggested that the anthocyanin synthesis pathway in Z. bungeanum is co-regulated by ZbMYB113 and $\mathrm{ZbHY} 5$. In addition, HY5 also regulated the flavonol biosynthesis in response to UVB [14]. This suggested that the role of UV-B-induced HY5 in flavonoid synthesis deserves further research.

Studies have found that HY5 regulates anthocyanin biosynthesis in two ways. On the one hand, it directly binds and induces the expression of some MYB transcription factor genes. On the other hand, it co-induces the expression of anthocyanin biosynthetic enzyme genes by interacting with other MYB factors [13]. In this study we focused on the interaction between $\mathrm{ZbHY} 5$ and $\mathrm{ZbMYB} 113$ and the result of BIFC also demonstrated the interaction between them. Furthermore, in the overexpression experiments, the expression level of ZbMYB113 increased 20-fold, far exceeding that of ZbHY5. This suggests that in addition to the interaction, $\mathrm{ZbHY} 5$ may also be a regulator of $\mathrm{ZbMYB113}$. This requires further research. MYB4 is a repressor of the anthocyanin synthesis pathway in response to UV-B. This may be the reason why the expression of some genes such as DFR decreased after some time. This is a good direction for the follow-up study of the UV-B-induced anthocyanin synthesis pathway.

\section{Materials and Methods}

\subsection{De Novo Assembly and Unigene Annotation}

All of the assembled unigenes were annotated by a sequence similarity search using BLAST (http:/ /blast.ncbi.nlm.nih.gov/Blast.cgi) (accessed on 13 September 2019) with an e-value $\leq 10^{-5}$ against other databases, including NR (ftp.ncbi.nlm.nih.gov/blast/db/ FASTA/nr.gz) (accessed on 20 September 2019), KOG, COG, eggNOG, KEGG, GO, and SwissProt [34]. Additionally, the predicted amino acid sequences of the unigenes were also mapped against the Pfam database using HMMER software (version 3.2.1) with an e-value $\leq 10^{-10}[35,36]$. Finally, the annotated information for the unigenes was obtained. Additionally, the automatic annotation of the flavonoid biosynthesis genes and the MYBs were used in this study $[37,38]$.

\subsection{Metabonomic Analysis and Transcriptome Analysis}

Z. bungeanum fruit was collected from eight-year-old trees grown in the experimental station of Northwest A\&F University, Shaanxi, Yangling, China. Fruit in the four stages of growth, S1 (green stage), S2 (light red stage), S3 (red stage), and S4 (deep red stage), were collected during the ripening process, and each sample had three biological replicates [39]. Fruit from each stage were divided into two groups: one for metabonomic flavonoid analysis and the other for transcriptome sequencing.

\subsection{The Treatment of Bagging Fruit}

About a month before maturity, the Z. bungeanum fruits were bagged in the field on 17 May 2020. After 20 d (6 June 2020), some of the bagged fruit ( 0 d) were collected for return to the laboratory, and the remaining bagged fruit were removed from the bags and collected after being exposed to natural light for $10 \mathrm{~d}$ (16 June 2020) and $20 \mathrm{~d}$ (26 June 2020) [18]. All the fruit came from different branches of the sunny side of the same tree, and they were all at a height of about $1.5 \mathrm{~m}$, which was convenient for bagging and picking. All the fruit were collected at 9:00-10:00 a.m. The samples were stored in a refrigerator at $-80{ }^{\circ} \mathrm{C}$ after being quick-frozen in liquid nitrogen for subsequent experiments.

\subsection{The Treatment of UV-B Irradiation for Leaves}

The leaves were collected from annual hydroponic seedlings of Z. bungeanum grown in a greenhouse. The annual Z. bungeanum seedlings were grown under continuous irradiation in Philips TL20W/01RS narrowband UV-B tubes $\left(1.5 \mathrm{mmol} \mathrm{m} \mathrm{m}^{-2} \mathrm{~s}^{-1}\right.$; measured with a VLX-3W Ultraviolet Light Meter equipped with a CX-312 sensor, Vilber Lourmat, Marne- 
laVallée, France). All the irradiated seedlings were darkened for $24 \mathrm{~h}$ in advance [40]. Then, the leaves were collected after $0 \mathrm{~h}, 3 \mathrm{~h}, 6 \mathrm{~h}, 12 \mathrm{~h}$, and $24 \mathrm{~h}$ of irradiation [18]. The samples were stored in a refrigerator at $-80{ }^{\circ} \mathrm{C}$ after being quick-frozen in liquid nitrogen for subsequent experiments.

\subsection{Bioinformatics Analysis of ZbbZIP Transcription Factor Family}

4.5.1. Excavation and Identification of ZbbZIP Transcription Factor Family Members

The transcriptional data related to anthocyanin biosynthesis in Z. bungeanum were screened from our previous transcriptome. The keywords "bZIP" and "basic leucine zipper" were searched directly in the transcriptome database, the two search results were summarized, and the repetitive sequences were deleted. The bZIP family protein sequence of the model plant Arabidopsis thaliana was downloaded from the TAIR database (http: / / web.arabidopsis.org) (accessed on 2 December 2020) as the query sequence, the blastp alignment (e-value set to 1e-5) was performed in the Z. bungeanum transcriptome data through the local BLAST+ software, and the repetitive sequences were deleted. Hidden Markov Models (HMMs) (PF00170, PF03131, and PF07716) of the conserved domains of the bZIP family were downloaded from the Pfam database (http://pfam.xfam.org) (accessed on 5 December 2020). The HMMs of the three bZIP domains were used as the query sequence to search the $Z$. bungeanum transcriptome data using the local HMMER 3.0 software; sequences whose e-values were less than $1 \mathrm{e}-5$ were kept, and the repetitive sequences were deleted. The common items in the sequences obtained by the above three methods were taken as the candidate protein sequences of the bZIP family in $Z$. bungeanum $[41,42]$.

The online software InterPro (http:/ / www.ebi.ac.uk/interpro/) (accessed on 10 December 2020) was used to identify and analyze the conserved domain of the candidate protein sequence, and the protein sequences not containing the bZIP domain were removed. The local MAFFT software was used to align all of the bZIP domain sequences and to manually check and delete the sequences with incomplete domains $[43,44]$.

\subsubsection{Construction of Phylogenetic Tree}

The local MAFFT software was used to perform multiple sequence alignments on the bZIP protein sequences of Z. bungeanum and Arabidopsis thaliana. The alignment results were imported into MEGA 7, and the neighbor joining (NJ) method was used to construct a phylogenetic tree. The parameter settings were as follows: bootstrap method 1000; Pdistance model; partial deletion; cutoff 50. Then, the online software ITOL was used to beautify the phylogenetic tree [41].

$\mathrm{ZbHY} 5$ was cloned, and the nucleotide sequence and amino acid sequence of $\mathrm{ZbHY} 5$ were obtained by gene sequencing. The amino acid sequences of the HY5 transcription factor in 40 kinds of plants (such as Arabidopsis thaliana, Citrus clementina, Malus domestica, etc.) were obtained from the NCBI database using BLASTP technology. MEGA 7 software was used to construct a phylogenetic tree [18].

\subsubsection{Differential Expression Pattern Analysis of ZbbZIP Transcription Factor Family Genes}

The FPKM values of the ZbbZIP transcription factor family genes were taken from the transcriptome, the logarithmic calculation method was used to perform hierarchical cluster analysis on the expression data of the ZbbZIP family genes, and Tbtools software was used to draw the expression heat map [41].

\subsubsection{Correlation Analysis of Anthocyanin Content and ZbbZIP Transcription Factor} Family Gene Expression

The FPKM values (greater than 20) of the ZbbZIP transcription factor family genes were taken in the transcriptome, and the total anthocyanin contents of the four growth and development stages in Z. bungeanum were taken from metabolome, and the local software $\mathrm{R}$ 3.6.1 was used to draw the correlation analysis heat map. 


\subsection{The Extraction and Measurement of Total Anthocyanins}

An amount of $0.15 \mathrm{~g}$ freeze-dried Z. bungeanum leaves or pericarps was weighed, quickly ground into powders in liquid nitrogen and soaked in $3 \mathrm{~mL}$ extraction solution (the volume ratio of methanol and hydrochloric acid was 97:3). After the samples were shaken and mixed, they were incubated in the refrigerator at $4{ }^{\circ} \mathrm{C}$ in the dark for $48 \mathrm{~h}$ and subsequently shaken and mixed once every $12 \mathrm{~h}$. Then, the mixed samples were ultrasonically extracted for $20 \mathrm{~min}$ and centrifuged at $6000 \mathrm{rpm}$ for $10 \mathrm{~min}$, and the supernatant was collected. Finally, with the extraction solution as a blank control, the absorbance of each sample at 530 and $657 \mathrm{~nm}$ was measured using an ultraviolet spectrophotometer. The total anthocyanin content $\left(\mu \mathrm{g} \cdot \mathrm{g}^{-1}\right)$ of each sample was calculated according to the following formula: $(\mathrm{A} 530-0.25 \times \mathrm{A} 657) \times 449.2 \times 3 \times 100 /(26,900 \times 0.15)[45]$.

\subsection{Extraction of Total RNA and Real-Time Fluorescence Quantitative PCR ( $q R T-P C R)$ Analysis}

The total RNA was extracted from the pericarps and leaves of $Z$. bungeanum using the Plant RNA Kit (Omega, R6827-01, Norcross, GA, USA). Then, 1000 ng RNA was used as the template to reverse-transcribe to the first-strand cDNA using the TransScript ${ }^{\circledR}$ OneStep gDNA Removal and cDNA Synthesis SuperMix Kit (TransGen, AE311-03, Beijing, China). Three biological replicates were set for each treatment. The Hieff qPCR SYBR Green Master Mix kit (High Rox) (Yeasen, 11203ES08, Shanghai, China) was used for the real-time fluorescent quantitative PCR (qRT-PCR) reaction. ZbUBQ was selected as the internal reference gene, and the cDNA obtained by reverse transcription was diluted five times and used as a template [46]. The primers for all of genes were designed using the Oligo 6 software and synthesized by Beijing Aoke Dingsheng Biotechnology Co., Ltd. All of the primers were used after being diluted 10 times. The reaction program was as follows: $95{ }^{\circ} \mathrm{C}$ pre-denaturation for $5 \mathrm{~min}, 95^{\circ} \mathrm{C}$ for $10 \mathrm{~s}, 58{ }^{\circ} \mathrm{C}$ for $20 \mathrm{~s}, 72{ }^{\circ} \mathrm{C}$ for $30 \mathrm{~s}, 40$ cycles. Each sample was set for three technical repetitions, and the results were analyzed using the $2^{-\Delta \Delta C T}$ method [47]. Genes such as ZbMYB113 and ZbbHLH35 were all identified by BLAST and phylogenetic tree analysis for qRT-PCR.

\subsection{Construction of Transient Gene Overexpression Vector}

The specific primers were designed and synthesized for transient gene overexpression. cDNA that had been reverse-transcribed from the RNA extracted from Z. bungeanum leaves exposed to UV-B for $6 \mathrm{~h}$ was used as a template. A $504 \mathrm{bp}$ full-length $\mathrm{ZbHY5}$ gene was obtained by PCR amplification using the Takara high-fidelity enzyme Primmer Star. The PCR program was set to pre-denaturation at $94{ }^{\circ} \mathrm{C}$ for $3 \mathrm{~min}$; denaturation at $98{ }^{\circ} \mathrm{C}$ for $10 \mathrm{~s}$; annealing at $57^{\circ} \mathrm{C}$ for $15 \mathrm{~s}$; extension at $72{ }^{\circ} \mathrm{C}$ for $10 \mathrm{~s}$ and 40 cycles; and extension at $72{ }^{\circ} \mathrm{C}$ for $10 \mathrm{~min}$. The target fragment was recovered. After adding the A tail, it was ligated to the cloning vector pMD19-T overnight at $4{ }^{\circ} \mathrm{C}$ to obtain the recombinant cloning vector ZbHY5-pMD19-T. The recombinant cloning vector was transferred into E. coli DH5 $\alpha$ competent cells and was used to extract the plasmid after verification through sequencing. At the same time, the pCambia2300 (referred to as $\mathrm{pC2300)} \mathrm{expression} \mathrm{vector} \mathrm{plasmid} \mathrm{was}$ extracted in the laboratory. Kpn1 and Sal 1 were used to double digest the ZbHY5-pMD19-T and $\mathrm{pC} 2300$ plasmids, and the T4 ligase was used to insert $Z b H Y 5$ into pC2300 to obtain a recombinant $Z b H Y 5$-pC2300 gene overexpression vector. The recombinant expression vector was transferred into $E$. coli $\mathrm{DH} 5 \alpha$ transcompetent cells. After verification, it was transferred to competent Agrobacterium tumefaciens GV3101 cells and stored in a refrigerator at $-80{ }^{\circ} \mathrm{C}$ for use [48].

\subsection{Transient Gene Overexpression Assay in Z. bungeanum Leaves}

An amount of $500 \mu \mathrm{L}$ of both the Agrobacterium tumefaciens pC2300 and ZbHY5-pC2300 broths were inoculated into $1 \mathrm{~mL}$ of LB liquid medium $\left(100 \mu \mathrm{L}\right.$ kanamycin of $50 \mathrm{mg} \cdot \mu \mathrm{L}^{-1}$ per $100 \mathrm{~mL}$ of LB medium) and activated in a shaker at $28^{\circ} \mathrm{C}$ for $12 \mathrm{~h}$. An amount of $1 \mathrm{~mL}$ activated bacterial solution was transferred to $20 \mathrm{~mL}$ of LB liquid medium (containing kanamycin) and incubated on a shaker at $28^{\circ} \mathrm{C}$ for $12 \mathrm{~h}$ to ensure that the $\mathrm{OD}_{600}$ of the 
bacterial solution reached approximately 2.0. The bacterial solution was centrifuged at $5000 \mathrm{rpm}$ at $4{ }^{\circ} \mathrm{C}$ for $5 \mathrm{~min}$, the bacterial cells were collected and resuspended in the resuspension solution (each $1 \mathrm{~L}$ resuspension solution contained $40 \mathrm{~mL} 250 \mathrm{mM}$ MES (2-morpholinoethanesulfonic acid) solution, $100 \mu \mathrm{L} 10 \mathrm{mM} \mathrm{MgCl} 2$ solution, and $3 \mathrm{~mL}$ $50 \mathrm{mM}$ AS (acetosyringone) solution). The $\mathrm{OD}_{600}$ of the resuspended bacterial solution was calculated and adjusted to about 1.0. The resuspended bacterial solution could be used for infection after being shaken in a shaker at $28^{\circ} \mathrm{C}$ at $100 \mathrm{rpm}$ for $3 \mathrm{~h}$ in the dark [48].

A disposable sterile syringe (with the needle removed) was used to inject the main leaf vein of the dorsum of the leaf for infection. After infection, the plants were placed in a light incubator for $24 \mathrm{~h}$ in low temperature and dark (temperature $10^{\circ} \mathrm{C}$; humidity $75 \%$; light intensity $0 \%$ ) conditions. UV-B light was used for induction (temperature $22{ }^{\circ} \mathrm{C}$; humidity $75 \%$; illuminance $0 \%$ ). The leaves were collected after $18 \mathrm{~h}$ of induction, quickly frozen in liquid nitrogen, and stored in a refrigerator at $-80{ }^{\circ} \mathrm{C}$ until their next use.

\subsection{Bimolecular Fluorescence Complementation (BiFC) Assay}

The ZbMYB113 gene was cloned with the specific primers. The full-length ZbHY5 and ZbMYB113 gene CDSs were, respectively, inserted into the C-terminal and the $\mathrm{N}$-terminal fragment of YFP to obtain ZbHY5-YFPC and ZbMYB113-YFP ${ }^{\mathrm{N}}$. The recombinant and empty plasmids were transformed into Agrobacterium tumefaciens GV3101 cells using the freeze-thaw method. The Agrobacterium tumefaciens cells were infiltrated into tobacco epidermal cells with a needleless syringe. After $48 \mathrm{~h}$, the YFP fluorescence signals were detected by a confocal laser scanning microscope [49].

\section{Conclusions}

The pericarp color greatly affects Z. bungeanum fruit quality. On the basis of the current results and those from previous studies, it can be concluded that ZbHY5 regulates the anthocyanin biosynthesis process through activating the transcription of related genes in response to light or UV-B irradiation. In addition, the $\mathrm{ZbHY} 5$ regulation process likely depends on interaction with ZbMYB113. Proper spacing between fruit trees can improve the utilization of light energy, thereby enhancing the expression level of the ZbHY5 gene. This finding suggests that high-quality fruit with a high anthocyanin content can be obtained by optimizing the spacing of fruit trees. In a word, this research provides evidence for the further investigation of the UV-B regulation of anthocyanin accumulation in Z. bungeanum.

Author Contributions: Conceptualization, H.L.; data curation, J.M. and R.C.; funding acquisition, D.W. and H.L.; methodology, J.Z. and J.M.; project administration, D.W.; resources, J.Z., S.Z. and C.W.; supervision, D.W.; validation, J.Z. and J.M.; visualization, S.Z. and R.C.; writing-original draft, J.Z. and J.M.; writing-review and editing, H.L. All authors have read and agreed to the published version of the manuscript.

Funding: This research was funded by National Key Research and Development Program of China, grant number 2019YFD1000600, and the National Natural Science Foundation of China, grant number 32171862 .

Institutional Review Board Statement: Not applicable.

Informed Consent Statement: Not applicable.

Data Availability Statement: The Illumina raw sequencing profiles were submitted to the NCBI BioProject database under number PRJNA807160.

Conflicts of Interest: The authors declare no conflict of interest.

\section{References}

1. Traka, M.H.; Mithen, R.F. Plant science and human nutrition: Challenges in assessing health-promoting properties of phytochemicals. Plant Cell 2011, 23, 2483-2497. [CrossRef] [PubMed]

2. Tanaka, Y.; Sasaki, N.; Ohmiya, A. Biosynthesis of plant pigments: Anthocyanin, betalains and carotenoids. Plant J. 2008, 54, 733-749. [CrossRef] [PubMed] 
3. Petroni, K.; Tonelli, C. Recent advances on the regulation of anthocyanin synthesis in reproductive organs. Plant Sci. 2011, 181, 219-229. [CrossRef]

4. Dubos, C.; Stracke, R.; Grotewold, E.; Weisshaar, B.; Martin, C.; Lepiniec, L. MYB transcription factors in Arabidopsis. Trends Plant Sci. 2010, 15, 573-581. [CrossRef]

5. Song, X.H.; Yang, Q.S.; Liu, Y.; Li, J.J.; Chang, X.C.; Xian, L.H. Genome-wide identification of Pistacia R2R3-MYB gene family and function characterization of PCMYB113 during autumn leaf coloration in Pistacia chinensis. Int. J. Biol. Macromol. 2021, 192, 16-27. [CrossRef] [PubMed]

6. Zhang, S.M.; Zhang, A.D.; Wu, X.X.; Zhu, Z.W.; Yang, Z.F.; Zhu, Y.L. Transcriptome analysis revealed expression of genes related to anthocyanin biosynthesis in eggplant (Solanum melongena L.) under high-temperature stress. BMC Plant Biol. $2019,19,387$. [CrossRef] [PubMed]

7. Lloyd, A.; Brockman, A.; Aguirre, L.; Campbell, A.; Bean, A.; Cantero, A. Advances in the MYB-bHLH-WD Repeat (MBW) Pigment Regulatory Model: Addition of a WRKY Factor and Co-option of an Anthocyanin MYB for Betalain Regulation. Plant Cell Physiol. 2017, 58, 1431-1441. [CrossRef] [PubMed]

8. Kami, C.; Lorrain, S.; Hornitschek, P.; Fankhauser, C. Light-regulated plant growth and development. Plant Dev. 2010, 91, 29-66.

9. Takos, A.M.; Jaffe, F.W.; Jacob, S.R.; Bogs, J.; Robinson, S.P.; Walker, A.R. Light induced expression of a MYB gene regulates anthocyanin biosynthesis in red apples. Plant Physiol. 2006, 142, 1216-1232. [CrossRef]

10. Xu, P.B.; Zawora, C.; Li, Y.; Wu, J.; Liu, L.C.; Liu, Z.C.; Cai, R.; Lian, H.L. Transcriptome sequencing reveals role of light in promoting anthocyanin accumulation of strawberry fruit. Plant Growth Regul. 2018, 86, 121-132. [CrossRef]

11. Cominelli, E.; Gusmaroli, G.; Allegra, D.; Galbiati, M.; Wade, H.K.; Jenkins, G.I.; Tonelli, C. Expression analysis of anthocyanin regulatory genes in response to different light qualities in Arabidopsis thaliana. J. Plant Physiol. 2008, 165, 886-894. [CrossRef] [PubMed]

12. Li, Y.; Xu, P.B.; Chen, G.Q.; Wu, J.; Liu, Z.C.; Lian, H.L. FvbHLH9, functions as a positive regulator of anthocyanin biosynthesis, by forming HY5-bHLH9 transcription complex in strawberry fruit. Plant Cell Physiol. 2020, 61, 826-837. [CrossRef] [PubMed]

13. Gangappa, S.N.; Botto, J.F. The multifaceted roles of HY5 in plant growth and development. Mol. Plant 2016, 9, 1353-1365 [CrossRef] [PubMed]

14. Stracke, R.; Favory, J.J.; Gruber, H.; Bartelniewoehner, L.; Bartels, S.; Binkert, M. The Arabidopsis bZIP transcription factor HY5 regulates expression of the PFG1/MYB12 gene in response to light and ultraviolet-B radiation. Plant Cell Environ. 2010, $33,88-103$

15. Favory, J.J.; Stec, A.; Gruber, H.; Rizzini, L.; Oravecz, A.; Funk, M.; Albert, A.; Cloix, C.; Jenkins, G.I.; Oakeley, E.J. Interaction of COP1 and UVR8 regulates UV-B-induced photomorphogenesis and stress acclimation in Arabidopsis. EMBO J. 2009, $28,591-601$. [CrossRef] [PubMed]

16. Zhang, H.Y.; He, H.; Wang, X.C.; Wang, X.F.; Yang, X.Z.; Li, L.; Deng, X.W. Genome-wide mapping of the HY5-mediated genenetworks in Arabidopsis that involve both transcriptional and post-transcriptional regulation. Plant J. 2011, 65, 346-358. [CrossRef]

17. Shin, J.; Park, E.; Choi, G. PIF3 regulates anthocyanin biosynthesis in an HY5-dependent manner with both factors directly binding anthocyanin biosynthetic gene promoters in Arabidopsis. Plant J. 2007, 49, 981-994. [CrossRef]

18. Wang, Y.Y.; Zhang, X.D.; Zhao, Y.R.; Yang, J.; He, Y.Y.; Li, G.C. Transcription factor PyHY5 binds to the promoters of PyWD40 and PyMYB10 and regulates its expression in red pear 'Yunhongli No. 1'. Plant Physiol. Biochem. 2020, 154, 665-674. [CrossRef]

19. Wolfgang, D.; Basten, L.S.; Berend, S.; Christoph, W. The Arabidopsis bZIP transcription factor family-An update. Curr. Opin. Plant Biol. 2018, 45, 36-49.

20. Zhang, Y.Q.; Zheng, S.; Liu, Z.J.; Wang, L.G.; Bi, Y.R. Both HY5 and HYH are necessary regulators for low temperature-induced anthocyanin accumulation in Arabidopsis seedlings. J. Plant Physiol. 2011, 168, 367-374. [CrossRef]

21. Qiu, Z.K.; Wang, H.J.; Li, D.J.; Yu, B.W.; Hui, Q.L.; Yan, S.S.; Huang, Z.J.; Cui, X.; Cao, B.H. Identification of Candidate HY5dependent and -independent Regulators of Anthocyanin Biosynthesis in Tomato. Plant Cell Physiol. 2019, 60, 643-656. [CrossRef] [PubMed]

22. Loyola, R.; Herrera, D.; Mas, A.; Wong, D.C.J.; Holl, J.; Cavallini, E.; Amato, A.; Azuma, A.; Ziegler, T.; Aquea, F.; et al. The photomorphogenic factors UV-B RECEPTOR 1, ELONGATED HYPOCOTYL 5, and HY5 HOMOLOGUE are part of the UV-B signalling pathway in grapevine and mediate flavonol accumulation in response to the environment. J. Exp. Bot. 2016, 67, 5429-5445. [CrossRef] [PubMed]

23. Huang, D.; Yuan, Y.; Tang, Z.Z.; Huang, Y.; Kang, C.Y.; Deng, X.X.; Xu, Q. Retrotransposon promoter of Ruby1 controls both light-and cold-induced accumulation of anthocyanin in blood orange. Plant Cell Environ. 2019, 42, 3092-3104. [CrossRef] [PubMed]

24. Dong, Y.H.; Beuning, L.; Davies, K.; Mitra, D.; Morris, B.; Kootstra, A. Expression of pigmentation genes and photo-regulation of anthocyanin biosynthesis in developing Royal Gala apple flowers. Aust J. Plant Physiol. 1998, 25, 245-252. [CrossRef]

25. Chattopadhyay, S.; Ang, L.H.; Puente, P.; Deng, X.W.; Wei, N. Arabidopsis bZIP protein HY5 directly interacts with light-responsive promoters in mediating light control of gene expression. Plant Cell 1998, 10, 673-683. [CrossRef] [PubMed]

26. Chang, C.S.; Li, Y.H.; Chen, L.T.; Chen, W.C.; Hsieh, W.P.; Shin, J.; Jane, W.N.; Chou, S.J.; Choi, G.; Hu, J.M.; et al. LZF1, a HY5-regulated transcriptional factor, functions in Arabidopsis de-etiolation. Plant J. 2008, 54, 205-219. [CrossRef] 
27. An, J.P.; Qu, F.J.; Yao, J.F.; Wang, X.N.; You, C.X.; Wang, X.F.; Hao, Y.J. The $b Z I P$ transcription factor MdHY5 regulates anthocyanin accumulation and nitrate assimilation in apple. Hortic. Res. 2017, 4, 17023. [CrossRef]

28. Liu, C.C.; Chi, C.; Jin, L.J.; Zhu, J.; Yu, J.Q.; Zhou, Y.H. The bZip transcription factor HY5 mediates CRY1a-induced anthocyanin biosynthesis in tomato. Plant Cell Environ. 2018, 41, 1762-1775. [CrossRef]

29. Wang, Y.L.; Wang, Y.Q.; Song, Z.Q.; Zhang, H.Y. Repression of MYBL2 by both microRNA858a and HY5 leads to the activation of anthocyanin biosynthetic pathway in Arabidopsis. Mol. Plant 2016, 9, 1395-1405. [CrossRef]

30. Jiang, Z.H.; Chen, C.; Wang, J.; Xie, W.Y.; Wang, M.; Li, X.S.; Zhang, X.Y. Purple potato (Solanum tuberosum L.) anthocyanin attenuate alcohol-induced hepatic injury by enhancing antioxidant defense. J. Nat. Med. 2016, 70, 45-53. [CrossRef]

31. Mcnellis, T.W.; Vonamim, A.G.; Araki, T.; Komeda, Y.; Miser, S.; Deng, X.W. Genetic and molecular analysis of an allelic series of cop1 mutants suggest functional roles for the multiple protein domains. Plant Cell 1994, 6, 487-500. [PubMed]

32. Shin, D.H.; Choi, M.; Kim, K.; Bang, G.; Cho, M.; Choi, S.B.; Choi, G.; Park, Y.I. HY5 regulates anthocyanin biosynthesis by inducing the transcriptional activation of the MYB75/PAP1 transcription factor in Arabidopsis. FEBS Lett. 2013, 587, $1543-1547$. [CrossRef] [PubMed]

33. Gonzalez, A.; Zhao, M.; Leavitt, J.M.; Lloyd, A.M. Regulation of the anthocyanin biosynthetic pathway by the TTG1/bHLH/Myb transcriptional complex in Arabidopsis seedlings. Plant J. 2008, 53, 814-827. [CrossRef]

34. Altschul, S.F.; Madden, T.L.; Schäffer, A.A. Gapped BLAST and PSI BLAST: A New Generation of Protein Database Search Programs. Nucleic Acids Res. Ital. 1997, 25, 3389-3402. [CrossRef] [PubMed]

35. Finn, R.D.; Bateman, A.; Clements, J. Pfam: The protein families database. Nucleic Acids Res. Ital. 2014, 42, D222-D230. [CrossRef]

36. Eddy, S.R. Profile hidden Markov models. Bioinform. Ital. 1998, 14, 755-763. [CrossRef]

37. Pucker, B. Automatic identification and annotation of $M Y B$ gene family members in plants. Biorxiv 2021. [CrossRef]

38. Pucker, B.; Reiher, F.; Schilbert, H.M. Automatic Identification of Players in the Flavonoid Biosynthesis with Application on the Biomedicinal Plant Croton tiglium. Plants 2020, 9, 1103. [CrossRef]

39. Sun, L.W.; Yu, D.M.; Wu, Z.C.; Wang, C.; Yu, L.; Wei, A.Z. Comparative Transcriptome Analysis and Expression of Genes Reveal the Biosynthesis and Accumulation Patterns of Key Flavonoids in Different Varieties of Zanthoxylum bungeanum Leaves. J. Agric. Food Chem. 2019, 67, 13258-13268. [CrossRef]

40. Zhao, B.B.; Wang, L.; Pang, S.Y.; Jia, Z.C.; Wang, L.; Li, W.X. UV-B promotes flavonoid synthesis in Ginkgo biloba leaves. Ind. Crops Prod. 2020, 151, 112483. [CrossRef]

41. Wang, Z.H.; Yan, L.Y.; Wan, L.Y.; Huai, D.X.; Kang, Y.P.; Shi, L. Genome-wide systematic characterization of bZIP transcription factors and their expression profiles during seed development and in response to salt stress in peanut. BMC Genom. 2019, 20, 51. [CrossRef] [PubMed]

42. Liu, J.Y.; Chen, N.N.; Chen, F.; Cai, B. Genome-wide analysis and expression profile of the $b$ ZIP transcription factor gene family in grapevine (Vitis vinifera). BMC Genom. 2014, 15, 281. [CrossRef] [PubMed]

43. Unel, N.M.; Cetin, F.; Karaca, Y.; Altunoglu, Y.C.; Baloglu, M.C. Comparative identification, characterization, and expression analysis of bZIP gene family members in watermelon and melon genomes. Plant Growth Regul. 2019, 87, 227-243. [CrossRef]

44. Wang, Z.H.; Cheng, K.; Wan, L.Y.; Yan, L.Y.; Jiang, H.F.; Liu, S.Y. Genome-wide identification and analysis of the basic leucine zipper (bZIP) transcription factor gene family in Ustilaginoidea virens. Genome 2017, 60, 1051-1059.

45. Liu, P.Y.; Wang, Y.L.; Meng, J.X.; Zhang, X.; Zhou, J.; Han, M.L. Transcriptome Sequencing and Expression Analysis of Genes Related to Anthocyanin Biosynthesis in Leaves of Malus ‘Profusion' Infected by Japanese Apple Rust. Forests 2019, 10, 665. [CrossRef]

46. Fei, X.T.; Shi, Q.Q.; Yang, T.X.; Fei, Z.X.; Wei, A.Z. Expression Stabilities of Ten Candidate Reference Genes for RT-qPCR in Zanthoxylum bungeanum Maxim. Molecules 2018, 23, 802. [CrossRef]

47. Schmittgen, T.D.; Livak, K.J. Analyzing real-time PCR data by the comparative C-T method. Nat. Protoc. 2007, 3, 1101-1108. [CrossRef]

48. Zhai, R.; Wang, Z.; Yang, C.; Wang, K.L.; Richard, E.; Liu, J.; Li, X.; Wu, Z.; Li, P.; Guan, Q.; et al. PbGA2ox8 induces vascular-related anthocyanin accumulation and contributes to red stripe formation on pear fruit. Hortic. Res. 2019, 6, 137. [CrossRef]

49. Zhang, H.; Gong, J.X.; Chen, K.L.; Yao, W.K.; Zhang, B.X.; Wang, J.Y. A novel R3 MYB transcriptional repressor, MaMYBx, finely regulates anthocyanin biosynthesis in grape hyacinth. Plant Sci. 2020, 298, 110588. [CrossRef] 